\title{
CREATING GENDER AFFIRMING INPATIENT PSYCHIATRIC CARE FOR TRANSGENDER TRANSITIONAL AGE YOUTH (TAY): NEW FRONTIERS IN CLINICAL CARE
}

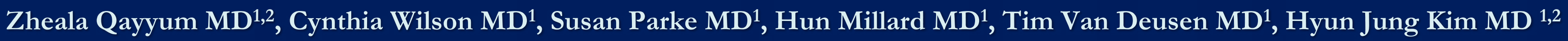

1) Yale School Of Medicine Department of Psychiatry 2) Harvard Medical School Department of Psychiatry

Introduction and Problem Addressed

-In 2015 the prevalence of transgender youth (TGD) in the general population ranges from $1 \%$ to $3.5 \%$ (3). These individuals faced unique challenges when seeking health care, as the system is often not prepared to manage issues that are common in this population (4).

-It is reported that transgender youth have a 2 to 3 -fold increased risk of depression, anxiety, self-injurious behavior, suicidal ideation, and suicide attempts compared with age-matched controls (5). Forty percent of transgender adults have attempted suicide, of whom $92 \%$ report attempting it before the age of 25 (1).

-It is critical that inpatient treatment settings be affirming and supportive, to avoid compounding trauma. Growing up in a culture where minority populations experience direct and indirect stigma, fear of rejection and discrimination, may lead to the creation of hostile environments. This minority stress has been linked to poorer health outcomes for TGD persons (2).

-Rejection from family and peers, prejudice, bullying and threats of violence all can contribute to worsening suicidal ideation and serious psychological distress (1).

\section{Statement of Project/Study Intent}

-Key ways in which hospital systems may create an affirming environment at the organizational level.

-Transforming inpatient units into affirming environments, and offering insight into effective staff development and training.

-Clinical considerations that may inform the care of TGD individuals in crisis.

\section{Description of Program/Intervention}

\begin{tabular}{|l|l|}
\hline \multicolumn{1}{|c|}{ Staff } & Development \\
\hline Case Studies & Multidisciplinary case conferences \\
\hline $\begin{array}{l}\text { Informal } \\
\text { Educational } \\
\text { Interventions }\end{array}$ & $\begin{array}{l}\text { Ad hoc conversations about pronoun } \\
\text { usage, current staff beliefs, staff skill } \\
\text { level in caring for TGD patients }\end{array}$ \\
\hline $\begin{array}{l}\text { Formal Educational } \\
\text { Interventions }\end{array}$ & $\begin{array}{l}\text { Nursing/Departmental grand rounds, } \\
\text { staff meetings, optional trainings }\end{array}$ \\
\hline $\begin{array}{l}\text { Continued } \\
\text { Education }\end{array}$ & $\begin{array}{l}\text { Existing platforms: weekly rounds, staff } \\
\text { meetings, case discussions }\end{array}$ \\
\hline
\end{tabular}
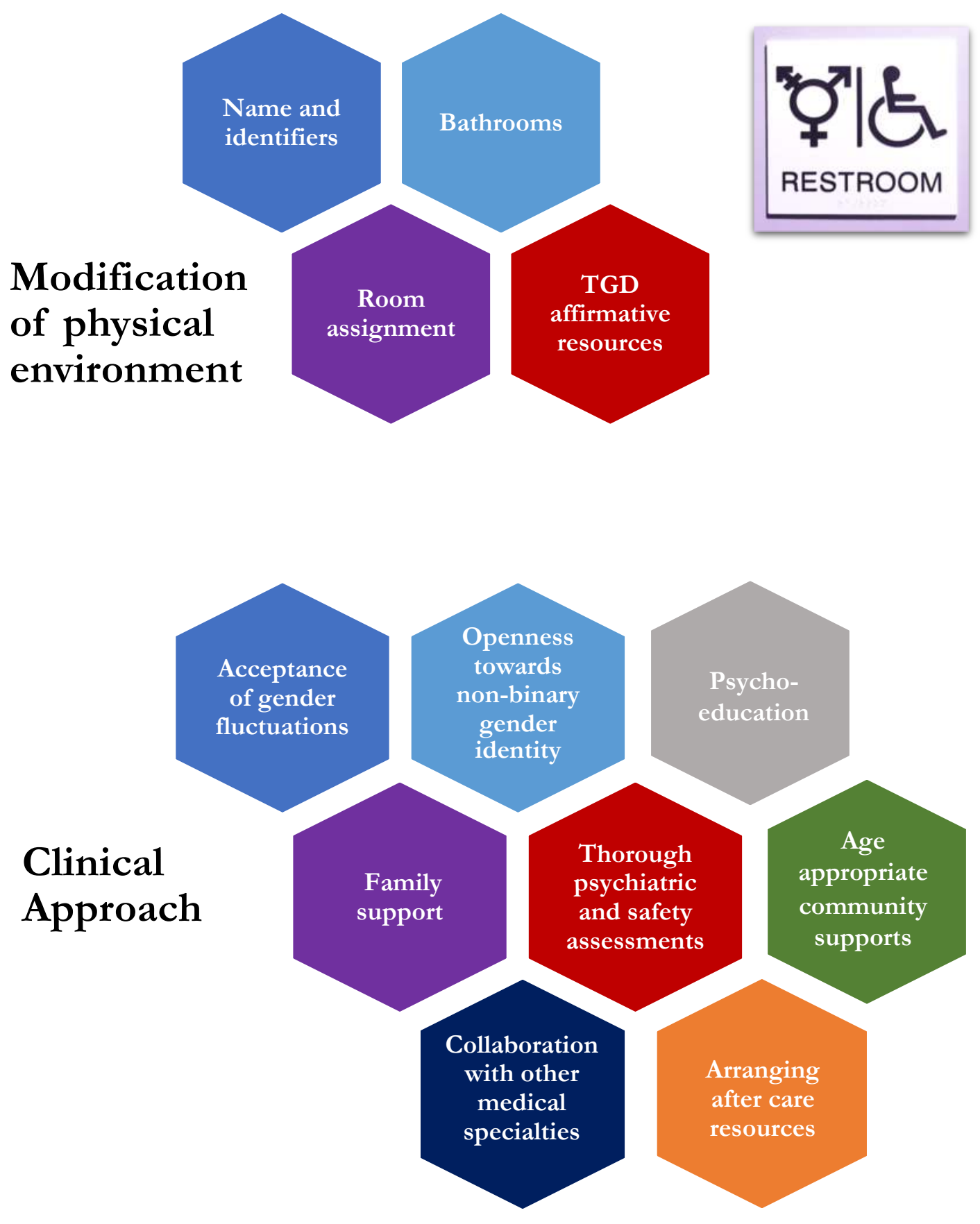

Key Lessons Learned

-Transgender and gender diverse youth are in a challenging position, as they may have greater needs for acute mental health treatment, but are less able to access this treatment in environments that are appropriately supportive.

-The literature highlights the need for greater education of mental health professionals in understanding and communicating with transgender individuals in clinical care settings.

-Although practical challenges exist, there are many opportunities to create small and large institutional changes benefitting these youth and families, educating and developing staff who have the necessary skills to treat mental health concerns, while supporting and affirming the dynamic process of gender exploration.

\section{Ongoing Challenges}

-Barriers: possible potentiation of stigma from peers and privacy concerns in communal living space.

-Personal Biases: of staff that persists despite adequate education.

-Intersectionality: where gender related concerns overlap with mental health concerns and psychosocial issues.

\section{References}

Please use the QR code to access the references. 